\title{
Vaccarin promotes endothelial cell proliferation in association with neovascularization in vitro and in vivo
}

\author{
FENGSHAN XIE $^{1}$, LEI FENG ${ }^{2}$, WEIWEI CAI ${ }^{1}$, YUYU QIU $^{2}$, YANLING LIU ${ }^{1}$, \\ YUE LI ${ }^{1}, \mathrm{BIN} \mathrm{DU}^{2}$ and LIYING QIU ${ }^{2}$ \\ ${ }^{1}$ Laboratory of Natural Medicine, School of Pharmaceutical Science; ${ }^{2}$ Wuxi Medical School, \\ Jiangnan University, Wuxi, Jiangsu 214122, P.R. China
}

Received June 4, 2014; Accepted January 7, 2015

DOI: $10.3892 / \mathrm{mmr} .2015 .3503$

\begin{abstract}
Angiogenesis is a major pathological component of several diseases, including traumatic vascular disease and coronary heart disease. The purpose of the present study was to determine the effects of vaccarin on endothelial cell migration and neovascularization, which are important and necessary components of wound healing. The present study investigated and confirmed neovascularization induced by vaccarin in vitro and in vivo. In vitro, the effects of vaccarin (1.08 and $2.15 \mu \mathrm{M})$ on proliferation, migration and tube formation of human microvascular endothelial cells (HMEC)-1 were evaluated via sulforhodamine B assay and migration and tube formation assay, respectively. Furthermore, a mouse Matrigel plus model was used to detect capillary-like tube structures in vivo. Immunohistochemistry was used to detect the protein expression of cluster of differentiation 31 (CD31), p-AKT and p-extracellular-signal-regulated kinases (Erk). Vaccarin significantly promoted HMEC-1 proliferation and migration and tube formation of HMEC-1 at a dose of $2.15 \mu \mathrm{M}$. In vivo, vaccarin delivered by daily oral administration significantly improved epidermal growth factor-induced angiogenesis in an intradermal inoculation mouse model. The mouse Matrigel model study also revealed that vaccarin significantly promoted neovascularization via detection of CD31 levels and enhanced protein expression of $\mathrm{p}$-Akt and p-Erk. In addition, vaccarin also promoted expression of CD31.
\end{abstract}

\section{Introduction}

Semen Vaccariae, the seeds of Vaccaria segetalis (Neck.) Garcke. ex Asch. (Caryophyllaceae), is a well-established traditional medicinal plant (1) used for activating blood circulation

Correspondence to: Professor Liying Qiu, Wuxi Medical School, Jiangnan University, 1800 Lihu Road, Wuxi, Jiangsu 214122, P.R. China

E-mail: qiulydoc@sina.com

Key words: vaccarin, human microvascular endothelial cell, proliferation, vasculogenesis, Matrigel plus model to promote menstruation, regulating menstrual disturbance and dispelling edema, promoting diuresis and milk secretion and relieving carbuncles $(2,3)$. The seeds contain flavonoids, cyclic peptides, triterpene saponins, lipids, aliphatic acids, monosaccharides, biotin and coumarin (4-10). A number of these compounds exhibit bioactive properties, including anti-angiogenic and growth-inhibitory activity on luteal cells, HL-60 cells and endothelial cells $(11,12)$. The present study investigated the effect of vaccarin from Semen vaccariae on human microvascular endothelial cells (HMEC)-1 in vitro in order to elucidate the efficacy of this medicine. Vaccarin (Fig. 1) is a major flavonoid glycoside found in Semen vaccariae. It is considered one of the major active constituents and is receiving increased attention (13).

A large number of studies have demonstrated that endothelial cells are important in the angiogenic process, including degradation of the extracellular matrix, migration, proliferation, tube formation and maturation $(14,15)$. The vascular endothelium is hypothesized to be critical in diabetes-associated vascular dysfunction, including atherosclerosis (16). Endothelial dysfunction leads to the pathogenesis of vascular diseases. Angiogenesis, the growth of new vasculature, is a critical biological response to ischemia, which provides biological revascularization of vascular obstructions (17). Therefore, promoting angiogenesis is a critical strategy and an area of growing interest in vascular disease therapeutics.

The promotional effects of vaccarin on angiogenic responses, including proliferation, migration and tube formation of HMEC-1 were assessed and confirmed. Phosphatidylinositol-4,5-bisphosphate 3-kinase (PI3K)/Akt and $\mathrm{RAF} / \mathrm{MEK} / \mathrm{extracellular-signal-regulated} \mathrm{kinase} \mathrm{(ERK)}$ are critical signaling pathways, which stimulate endothelial cell proliferation, migration and tube formation (18). In vivo, the effects of angiogenesis induced by Matrigel plug were investigated in a mouse model.

\section{Materials and methods}

Drugs and chemicals. Vaccarin was purchased from Shanghai Shifeng Technology Co., Ltd. (Shanghai, China). The HMEC-1 cell line was obtained from the French Institute of Health and Medical Research (Paris, France). Matrigel ${ }^{\mathrm{TM}}$ was purchased from Becton-Dickinson (Oxford, UK). MCDB-131, 
rhodamine B, epidermal growth factor (EGF), fetal bovine serum (FBS) and trypsin were purchased from Sigma-Aldrich (St. Louis, MO, USA). CD31 rabbit polyclonal antibody was purchased from Sangon Biotech (Shanghai) Co., Ltd. (cat. no. AB60701a; Shanghai, China). P-Erk (ab76165) and p-Akt (ab66138) rabbit polyclonal antibodies were purchased from Abcam (Hong Kong, China). Goat anti-rabbit secondary immunoglobulin $(\mathrm{Ig}) \mathrm{G}$ antibody was purchased from Sangon Biotech (Shanghai) Co., Ltd. The SABC kit and Masson dye were obtained from Nanjing Jiancheng Biological Technology, Inc. (Nanjing, China).

Cell culture and treatments. HMEC-1 cells were cultured in MCDB-131 medium supplemented with 10\% FBS, 2 mM L-glutamine, $100 \mathrm{U} / \mathrm{ml}$ penicillin and $100 \mathrm{~g} / \mathrm{ml}$ streptomycin at $37^{\circ} \mathrm{C}$ in $5 \% \mathrm{CO}_{2}$. After the treatments were administered, the cells were harvested for further analysis.

Analysis of cell proliferation. The effects of vaccarin on HMEC-1 growth promotion were measured using a sulforhodamine B (SRB) assay. Briefly, adherent cells were seeded into each well of 96 -well plates and cultured at $37^{\circ} \mathrm{C}$ in a $5 \%$ $\mathrm{CO}_{2}$ saturated humidity condition for $24 \mathrm{~h}$, then incubated with different concentrations of vaccarin for $48 \mathrm{~h}$. Following removal of the culture medium (10\% FBS), $200 \mu 1$ trichloroacetic acid solution was added into each well at $4^{\circ} \mathrm{C}$ for $40 \mathrm{~min}$. Subsequently, the mixture was cleaned and dried at $37^{\circ} \mathrm{C}$, then $100 \mu \mathrm{l} \mathrm{SRB}$ was added into each well for $20 \mathrm{~min}$. Following washing, $150 \mu \mathrm{l}$ Tris solution was added into each well. The mixture was agitated and measured at $540 \mathrm{~nm}$ using a Multiskan MK3 microplate reader (Thermo LabSystems, Inc., Milford, MA, USA) and the cell viability was expressed as an optical density. The control was defined as $100 \%$. The average level was calculated using the proliferation rate formula: Percent proliferation $=$ Test well signal $/$ Control group signal x $100 \%$.

Cell migration assay. Briefly, HMEC-1 cells $\left(8 \times 10^{3}\right.$ cells/well) were seeded onto each well and cultured at $37^{\circ} \mathrm{C}$ in a saturated humidity containing $5 \% \mathrm{CO}_{2}$ for $24 \mathrm{~h}$. Once the cells had completely attached, the middle of the cell plate was scraped with a line $\sim 1 \mathrm{~mm}$ wide and then changed to MCDB medium containing 10\% FBS and two different concentrations of vaccarin $(1.08$ and $2.15 \mu \mathrm{M})$. The cells were incubated and images were captured of randomly selected fields (magnification, $\mathrm{x} 100$ ) under a microscope video system (Olympus IX70; Olympus, Tokyo, Japan). The width of each gap in the three different sections was measured and averaged.

Capillary tube formation assay. The capillary tube formation assay was performed as described previously (19). Briefly, the 96 well plate was coated with $0.1 \mathrm{ml}$ Matrigel and the material was allowed to solidify and polymerize at $37^{\circ} \mathrm{C}$ for $30 \mathrm{~min}$. Each well was seeded with $4 \times 10^{5}$ HMEC-1 cells and incubated in MCDB medium containing 10\% FBS and different concentrations of vaccarin $(1.08$ and $2.15 \mu \mathrm{M})$ for $6,12,24$ and $48 \mathrm{~h}$. Subsequently, images were captured of the tubular structures from five randomly selected fields using an inverted fluorescence microscope (Olympus IX70; Olympus) at different time points.

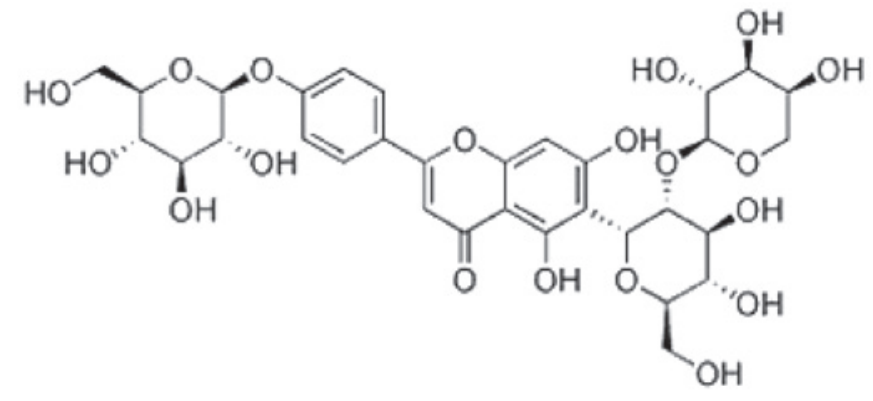

Figure 1. Chemical structure of vaccarin.

Establishment of mouse matrigel plug model and treatment. The present study was approved by the ethics committee of Jiangnan University (JN no. 20130327-0702; Jiangsu, China) and was conducted in accordance with the guidelines issued by the State Food and Drug Administration (Beijing, China). The Matrigel plug assay in imprinting control region (ICR) mice was performed as described previously with specific modifications (20). Female ICR mice, aged 4-5 weeks old and weighing 17-20 g, were subcutaneously injected with $500 \mu \mathrm{l}$ of Matrigel containing heparin (113 units) and mouse EGF (120 ng) into the left hypogastric region. Matrigel mixed with heparin alone was included as a blank control. The next day following implantation of Matrigel, mice were orally administered a single dose of saline. In addition, mice were divided into four groups (six mice per group), two groups were treated with oral vaccarin at doses of 5 and $10 \mathrm{mg} / \mathrm{kg} /$ day, respectively. The EGF control and blank control groups were treated with saline. After two weeks, the Matrigel plugs were carefully removed and images were captured. They were then formalin-fixed and paraffin-embedded. To determine the number of endothelial cells, hematoxylin and eosin (H\&E) and Masson's Trichrome (MT) staining were performed to visualize endothelial infiltration. Briefly, $5 \mu \mathrm{m}$ sections were stained with H\&E and MT solution. The number of blood vessels in the high power field was quantified by counting vessel infiltration in the plugs.

Immunohistochemistry. Matrigel tissue sections of the same volume were placed onto treated slides. According to sequences, sections were fixed, deparaffinized and rehydrated in distilled water. Tissues were boiled in $2 \%$ citrate buffer at $95^{\circ} \mathrm{C}$ for $20 \mathrm{~min}$, then treated with $3 \%$ hydrogen peroxide in order to block the activity of endogenous peroxidase. The slides were incubated with protein-blocking agent, then treated with the primary antibodies, including CD31 (1:25), p-Erk (1:40) and p-Akt (1:40) at $4^{\circ} \mathrm{C}$ overnight. The tissues were then incubated with the secondary biotinylated IgG antibodies $(1: 2,000)$ and finally stained with 3,3'-diaminobenzidine according to the manufacturer's instructions (EnoGene Biotech Co., Ltd., New York, NY, USA). Counterstaining was performed with hematoxylin. Microvascular density (MVD) was determined, as specified by Weidner (21). p-Erk and p-Akt expression was determined using Image-Pro Plus 6.0 software (Media Cybernetics, Rockville, MD, USA).

Statistical analysis. The results are presented as the mean \pm standard deviation. Statistical analysis was performed using analysis of variance/Dunnett's t-test for multiple group 
comparisons and Student's t-test for two group comparisons using SPSS 20.0 software (SPSS, Inc., Chicago, IL, USA). $\mathrm{P}<0.05$ was considered to indicate a statistically significant difference.

\section{Results}

Effects of vaccarin on cell proliferation. The effects of vaccarin on HMEC-1 cells were evaluated. The results revealed that vaccarin increased the proliferative activity of HMEC-1 cells. The optimum concentration of vaccarin was $2.15 \mu \mathrm{mol} / \mathrm{l}^{-1}$ (proliferation ratio / $125.62 \pm 1.14 \%$ of control; $\mathrm{P}<0.01$ ). The proliferation ratio of vaccarin at $0.54,1.08,4.30,8.60,17.20$ and $34.40 \mu \mathrm{M}$ was $103.15 \pm 0.95,109.71 \pm 0.85,117.54 \pm 1.65$, $114.54 \pm 1.85$ and $110.21 \pm 1.27 \%$ of the control, respectively ( $<<0.05$; Fig. 2A).

Vaccarin promotes HMEC-1 migration and tube formation. As shown in Fig. 2B and C, the effects of vaccarin on HMEC-1 migration and tube formation in vitro were analyzed. It was demonstrated that vaccarin significantly promoted migration, when treated with concentrations of 1.08 and $2.15 \mu \mathrm{M}$, compared with the control group, for 24 and $48 \mathrm{~h}$, respectively (Fig. 2D). As shown in Fig. 2C and Db, the control group did not form a complete, enclosed and robust network of tubes at $6 \mathrm{~h}$, however, vaccarin stimulated the angiogenic differentiation of HMEC-1 cells (tube formation) on Matrigel at the same time at doses of $2.15 \mu \mathrm{M}(\mathrm{P}<0.01)$. Furthermore, after 24 and $48 \mathrm{~h}$, the tube formation numbers of the vaccarin groups significantly increased compared with those of the control groups $(\mathrm{P}<0.05)($ Fig. $2 \mathrm{Db})$; however, the group treated with $2.15 \mu \mathrm{M}$ vaccarin maintained a complete, enclosed and strong network of tubes as shown in Fig. 2C.

Vaccarin promotes EGF-induced blood vessel formation in mice. To examine whether the angiogenic function of vaccarin exhibits a response to neovascularization activity in vivo, Matrigel allografts were implanted subcutaneously into ICR mice to establish an angiogenesis assay in vivo (22). As shown in Fig. 3A, Matrigel plugs containing EGF alone were light red, however, the EGF with oral treatment of vaccarin $(5 \mathrm{mg}$ and $10 \mathrm{mg} / \mathrm{kg} /$ day) were dark red, particularly the higher dose. This suggested that functional vasculature had formed in the EGF group and a greater density of blood vessels were present in the Matrigel plugs of mice subjected to oral vaccarin treatment. To confirm this conclusion, the number of endothelial cells in the Matrigel plugs were compared using MT and H\&E staining. MT stained the Matrigel blue and the endothelial cells/vessels red and H\&E stained cells purple and the Matrigel pink (Fig. 3A). More vessels and endothelial cells were observed in the Matrigel plugs treated with EGF and vaccarin than in those plugs treated with EGF alone (Fig. 3A, 3Ca and b). The results demonstrated that vaccarin at concentrations of 5 and $10 \mathrm{mg} / \mathrm{kg}$ significantly enhanced EGF-induced angiogenesis after 14 days of Matrigel implantation and this was consistent with the results of the tube formation assay.

To further examine whether vaccarin may promote angiogenesis, Matrigel tissues were stained with antibodies against CD31, p-Erk and p-Akt (Fig. 3B). An increased number of vessels and endothelial cells were observed in the Matrigel
A

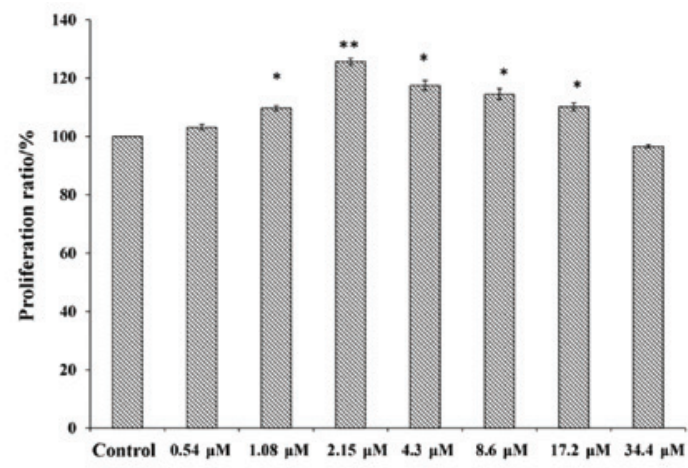

B
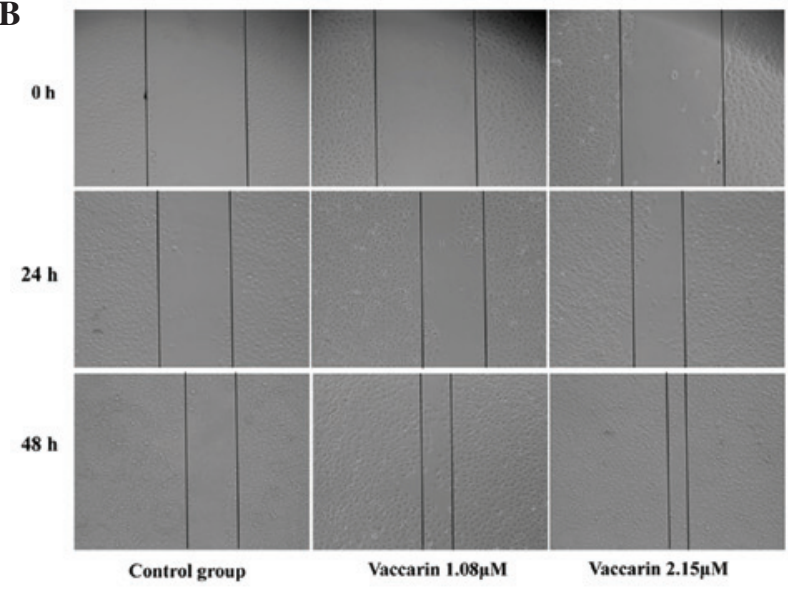

C

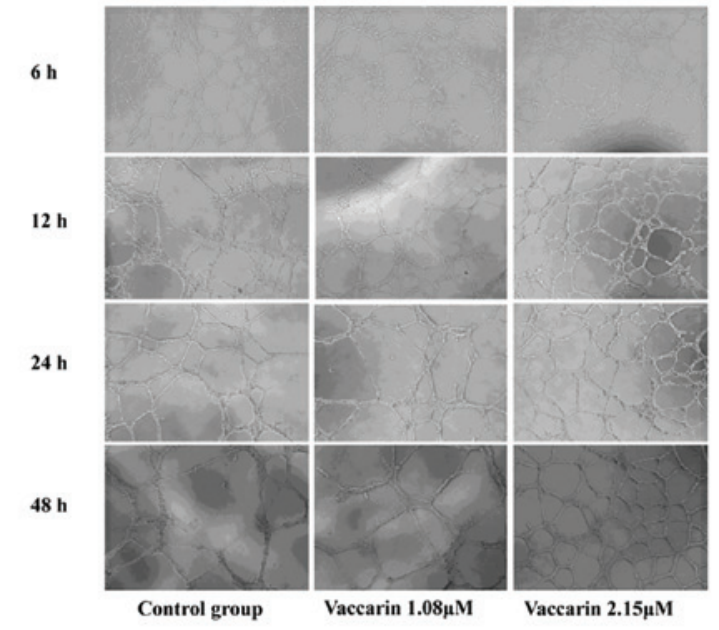

D a

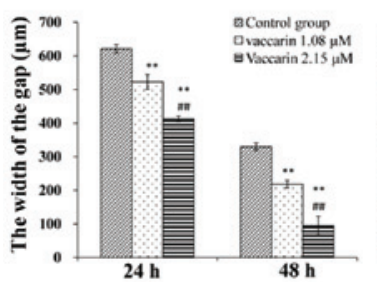

b
Figure 2. Effects of vaccarin on proliferation, migration and tube formation in HMEC-1 cells ( $n=6)$. (A) HMEC-1 cells were cultured for $48 \mathrm{~h}$ with different concentrations of vaccarin. (B) Migration was analyzed by averaging the width of each gap in six places. (C) Tube formation image of HMEC-1 cells with vaccarin treatment at different concentrations for different time periods. (magnification, x100; staining, hematoxylin and eosin). (Da) Analysis of migration width of gaps; (Db) analysis of the number of tubes formed. Data are presented as the mean \pm standard error of the mean from six independent experiments. ${ }^{*} \mathrm{P}<0.05,{ }^{* *} \mathrm{P}<0.01$ compared with the control group; ${ }^{\# \#} \mathrm{P}<0.01$ compared with the vaccarin $1.08 \mu \mathrm{M}$ group. HMEC, human microvascular endothelial cells. 
A

a

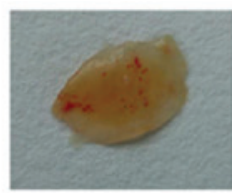

b

HE
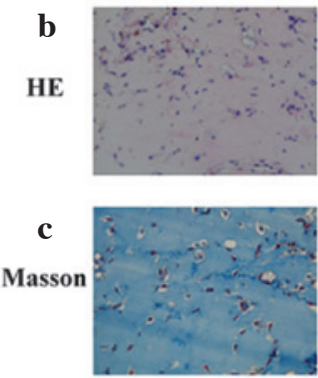

Blank control
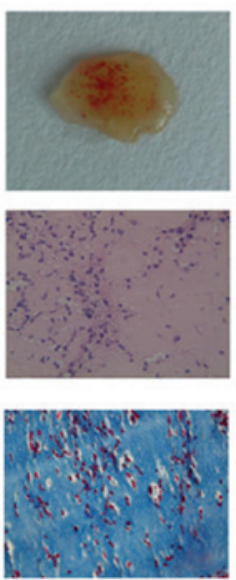

EGF control
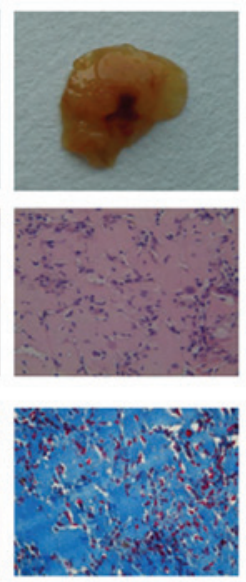

EGF+5 mg vaccarin
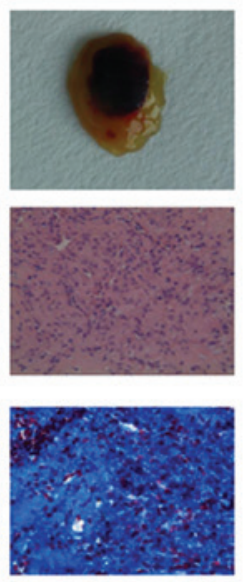

EGF+10 mg vaccarin

B

CD31
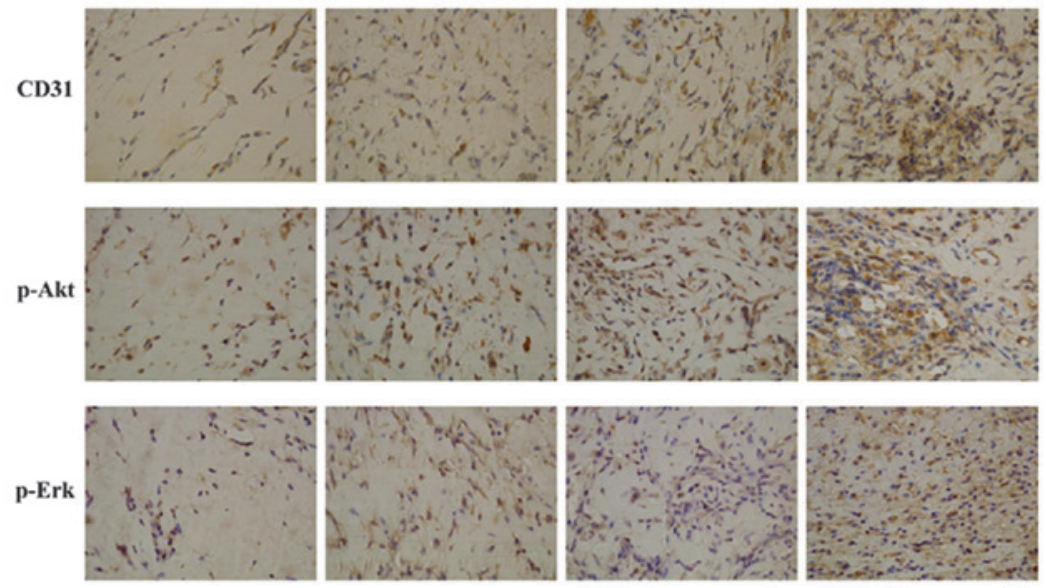

Blank control

EGF control

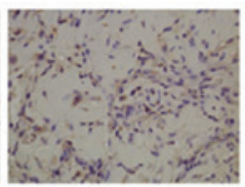

EGF+5 mg vaccarin

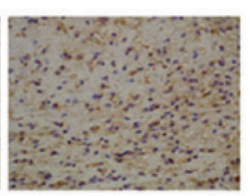

EGF+10 mg vacearin

C
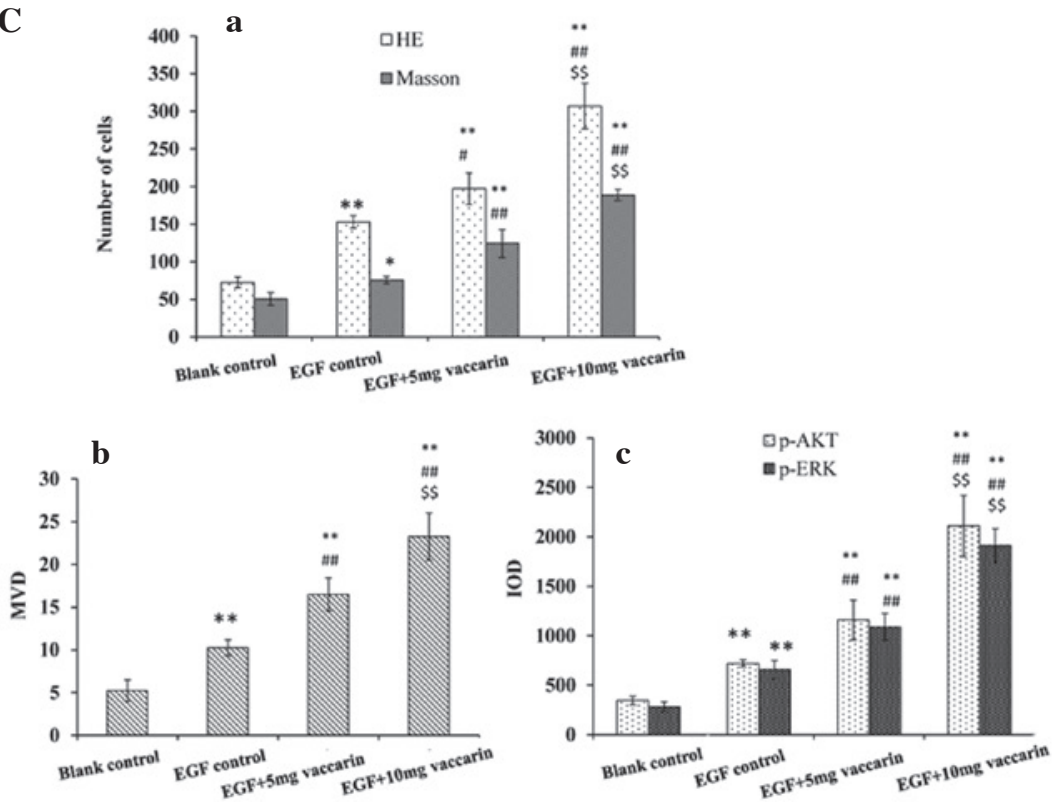

Figure 3. Effect of vaccarin on angiogenesis using a murine Matrigel plug assay ( $\mathrm{n}=6$ ). (Aa) Vaccarin promoted angiogenesis induced by EGF in the murine Matrigel plug model; ( $\mathrm{Ab}$ ) serial sections $(5 \mu \mathrm{m})$ were processed for hematoxylin and eosin staining (magnification, $\mathrm{x} 400$ ); (Ac) representative MT staining of Matrigel plug from each group (magnification, $\mathrm{x} 400)$. (B) Serial sections $(5 \mu \mathrm{m})$ were processed for immunohistochemistry (magnification, $\mathrm{x} 400)$ with antibodies against CD31, p-Akt and p-Erk. (Ca) Compared with the blank control and EGF control groups, the number of endothelial cells were significantly higher following treatment with vaccarin through MT and $\mathrm{H} \& \mathrm{E}$ staining. $(\mathrm{Cb})$ Effect of vaccarin on the angiogenesis of transplantable Matrigel was evaluated by MVD/CD31 compared with the blank control and EGF control groups. (Cc) Compared with the blank control and EGF control groups, p-Akt and p-Erk expression was higher following treatment with vaccarin. Data are presented as the mean \pm standard error of the mean from six independent experiments. ${ }^{*} \mathrm{P}<0.05,{ }^{* *} \mathrm{P}<0.01$ compared with the blank control group; ${ }^{\# \prime} \mathrm{P}<0.01$, compared with the EGF control group; ${ }^{\$ \$} \mathrm{P}<0.01$, compared with the EGF+5 mg vaccarin group. MT, Masson's Trichrome; EGF, epidermal growth factor; H\&E, hematoxylin and eosin; Erk, extracellular signal-related kinase; MVD, microvascular density; IOD, integrated optical density; CD31, cluster of differentiation 31. 
plugs treated with both EGF and vaccarin compared with those plugs treated with EGF alone (Fig. 3A, 3Ca and 3Cb). These results suggested that vaccarin at the concentrations of 5 and $10 \mathrm{mg} / \mathrm{kg}$ remarkably enhanced the EGF-induced angiogenesis following 14 days of matrigel implantation; in addition, these results are consistent with the results of tube formation assay.

The neovascularization activity was measured based upon the MVD via detecting expression of CD31. Compared with the controls, the MVD of vaccarin-treated groups at concentrations of 5 and $10 \mathrm{mg} / \mathrm{kg}$ exhibited a significant increase (Fig. 3Cb). In addition, activation of p-Erk and p-Akt has been verified to correlate with angiogenesis (23). It was found that vaccarin treatment markedly upregulated the expression of p-Erk and p-Akt in blood vessels (Fig. 3Cc), further indicating that vaccarin was able to promote angiogenesis, possibly through the MAPK/Erk and PI3K/Akt signaling pathways.

\section{Discussion}

The endothelium is critical in the regulation of vascular function and the development of physiological and pathophysiological inflammation. Evidence exists to support the concept that a graft lined with a significant number of endothelial cells may consequently lead to an improved graft survival rate (24). Using natural medicine to treat various diseases is a long tradition in China. The present study focused on examining a potential angiogenic compound based upon its usage in traditional medicine.

Semen vaccariae has been used to treat various diseases for thousands of years (1), however, the mechanism underlying its promotional effects on angiogenesis remain to be elucidated. Previous studies have reported that the chemical composition of Semen vaccariae includes flavonoids, cyclic peptides, triterpene saponins, lipids, aliphatic acids, monosaccharides, biotin and coumarin (4-10). A number of these compounds exhibit anti-angiogenic and growth-inhibitory activity on different cells $(6,7,25,26)$. The present study focused on the stimulating effects of vaccarin on HMCE-1 proliferation, migration and tube formation, which are key characteristics of endothelial cells in angiogenesis. Vaccarin has been demonstrated to positively affect cellular proliferation in the process of angiogenesis in HMEC- 1 cells. The present study observed that vaccarin at a dose of $2.15 \mu \mathrm{mol} / \mathrm{l}^{-1}$ increased the proliferation of HMEC-1 cells, following cultivation for $48 \mathrm{~h}$. In the present study, vaccarin significantly stimulated HMEC-1 migration, which is an important and early event in the angiogenic process. The stimulating effects of vaccarin in vitro were evaluated using the wound healing assay and a two-dimensional Matrigel model. A tube formation assay is a widely used model in order to assess angiogenic activity (27). In the two models, vaccarin promoted the cell migration and capillary tube formation of HMEC-1 cells, suggesting that vaccarin increased angiogenesis by accelerating the formation of cellular connections.

In order to investigate the effect of vaccarin on the promotion of angiogenesis in vivo, mouse models were injected with Matrigel. Following intragastric administration of vaccarin each day, the quantity of blood vessels in matrigel implants markedly increased compared with the control group. MVD is a marker to assess the level of angiogenesis and an increase of MVD in Matrigel tissue suggests fast-growing blood vessels. Immunohistochemical staining with antibodies against CD31 revealed that the MVD significantly increased in the vaccarin-treated group. Following treatment with vaccarin in vivo, its stimulating effect on angiogenesis was correlated with increased MVD. The MVD was significantly increased at concentrations of 5 and $10 \mathrm{mg} / \mathrm{kg}$ administered daily.

Angiogenesis stimulated by EGF is a complex process. EGF acts as a potent angiogenic agent that exerts its effect on cells through a transmembrane receptor with tyrosine kinase activity, which in turn activates a variety of intracellular signaling cascades to regulate cellular functions. PI3K and its downstream activated serine/threonine kinase Akt are involved in several processes of angiogenesis, including endothelial cell migration, proliferation and survival (28). The PI3K/Akt pathway and RAF/MEK-ERK pathway are involved in EGF-induced angiogenesis. The phosphorylation of EGFR is required for the activation of Akt, which contributes to the proliferation and survival of endothelial cells. In addition, the phosphorylation of EGFR activates MAPK/ERK cascades, which also promote the proliferation and migration of endothelial cells $(23,28)$. In addition, the results from immunohistochemical staining revealed that the expression of $\mathrm{p}-\mathrm{Akt}$ and p-Erk was significantly increased in the vaccarin-treated group. These signaling molecules are the main factors associated with the EGF signaling pathway in angiogenesis of vascular endothelial cells. It is noteworthy that vaccarin was potent when administered alongside a vascular disease treatment, which correlated with angiogenesis.

In conclusion, vaccarin promoted endothelial cell proliferation, migration and tube formation in vitro and accelerated angiogenesis in vivo. Based on the current results, vaccarin may be used as a potent angiogenic and vascular disease treatment.

\section{Acknowledgements}

This study was supported by a grant from the Fundamental Research Funds for the Central Universities (grant no. JUSRP51412B).

\section{References}

1. China Pharmacopoeia Committee: Chinese Pharmacopoeia. Vol. 1. 2010 edition. China Medical Science Press, Beijing, China, pp49-50, 2010.

2. Sang SM, Lao A, Chen ZL, Uzawa J and Fujimoto Y: Chemistry and bioactivity of the seeds of Vaccaria segetalis. In: Oriental Foods and Herbs: Chemistry and Health Effects. Vol 859. Ho C, Lin J and Zheng QY (eds). American Chemical Society, Washington DC, WA, pp279-291, 2003.

3. Li F and Liang JY: Research progress of Vaccaria segetalis. Straits Pharm J 3: 1-5, 2007.

4. Koike K, Jia Z and Nikaido T: Triterpenoid saponins from Vaccaria segetalis. Phytochemistry 47: 1343-1349, 1998.

5. Sang SM, Lao AN and Wang HC: A phenylpropanoid glycoside from Vaccaria segetalis. Phytochemistry 48: 569-571, 1998.

6. Sang S, Lao A, Wang H, et al: Triterpenoid saponins from Vaccaria segetalis. J Asian Nat Prod Res 1: 199-205, 1999.

7. Yun YS, Morita H, Takeya K, et al: Cyclic peptides from higher plants. 34. segetalins $G$ and $H$, structures and estrogen-like activity of cyclic pentapeptides from Vaccaria segetalis. J Nat Prod 60: 216-218, 1997.

8. Morita H, Yun YS, Takeya K, et al: A cyclic heptapeptide from Vaccaria segetalis. Phy tochemistry 42: 439-441, 1996. 
9. Dong Z and Chen N: Study on the TLC identification method of Wangbuliuxing tablets. China For Med Treat 30: 128, 2011 (In Chinese)

10. Li N, Ma CH, Liu D, et al: Chemical constituents analysis of fried Vaccaria segetalis. Chin J Exptl Tradit Med Form 19: 73-75, 2013.

11. Sun Y, Liang H, Zhang X, et al: Structural elucidation and immunological activity of a polysaccharide from the fruiting body of Armillaria mellea. Bioresour Technol 100: 1860-1863, 2009.

12. Sun Y, Wang S, Li T, et al: Purification, structure and immunobiological activity of a new water-soluble polysaccharide from the mycelium of Polyporus albicans (Imaz) Teng. Bioresour Technol 99: 900-904, 2008.

13. Meng H, Chen Y, Qin W, et al: Determination of vaccarin in Vaccariae Semen by HPLC. Zhongguo Zhong Yao Za Zhi 35: 2072-2074, 2010 (In Chinese).

14. Folkman J: Angiogenesis. Annu Rev Med 57: 1-18, 2006.

15. Folkman J: Angiogenesis: an organizing principle for drug discovery? Nat Rev Drug Discov 6: 273-286, 2007.

16. Vita JA: Endothelial function and clinical outcome. Heart 91: 1278-1279, 2005.

17. Rosengart TK, Patel SR, Crystal RG: Therapeutic angiogenesis: protein and gene therapy delivery strategies. J Cardiovasc Risk 6 : 29-40, 1999.

18. Kang Z, Jiang W, Luan H, Zhao F and Zhang S: Cornin induces angiogenesis through PI3K-Akt-eNOS-VEGF signaling pathway. Food Chem Toxicol 58: 340-346, 2013.

19. Min JK, Han KY, Kim EC, et al: Capsaicin inhibits in vitro and in vivo angiogenesis. Cancer Res 64: 644-651, 2004.
20. Malinda KM: In vivo matrigel migration and angiogenesis assay. Methods Mol Biol 467: 287-294, 2009.

21. Weidner N: Current pathologic methods for measuring intratumoral microvessel density within breast carcinoma and other solid tumors. Breast Cancer Res Treat 36: 169-180, 1995.

22. Adini A, Fainaru O, Udagawa T, Connor KM, Folkman J and D'Amato RJ: Matrigel cytometry: a novel method for quantifying angiogenesis in vivo. J Immunol Methods 342: 78-81, 2009.

23. Takeuchi K, Yanai R, Kumase F, Morizane Y, Suzuki J, Kayama M, et al: EGF-like-domain-7 is required for VEGF-induced Akt/ERK activation and vascular tube formation in an ex vivo angiogenesis assay. PLoS One 9: e91849, 2014.

24. Kakisis JD, Liapis CD, Breuer C, et al: Artificial blood vessel: the holy grail of peripheral vascular surgery. J Vasc Surg 41: 349-354, 2005.

25. Sang SM, Lao AN, Leng Y, et al: A new triterpenoid saponin with inhibition of luteal cell from the seeds of Vaccaria Segetalis. J Asian Nat Prod Res 4: 297-301, 2002.

26. Sang SM, Lao AN, Chen ZL, et al: Three new triterpenoid saponins from the seeds of Vaccaria Segetalis. J Asian Nat Prod Res 2: 187-193, 2000.

27. Goodwin AM: In vitro assays of angiogenesis for assessment of angiogenic and anti-angiogenic agents. Microvasc Res 74: $172-183,2007$.

28. Kowanet M and Ferrara N: Vascular endothelial growth factor signaling pathways: Therapeutic perspective. Clin Cancer Res 12: 5018-5022, 2006. 